\title{
Paper A Novel System Architecture for an Improved Self-care Solution - Conceptual Design and Key Components
}

\author{
Patrick Santos, Jorge Ramos, Eduardo Seabra, and José Castro \\ Uptec Parque da Ciencia e da Tecnologia da Universidade do Porto, Porto, Portugal
}

https://doi.org/10.26636/jtit.2020.142920

\begin{abstract}
The high penetration rate that mobile devices enjoy in to day's society has facilitated the creation of new digital services, with those offered by operators and content providers standing out. However, even this has failed to encourage consumers to express positive opinions on telecommunication services, especially when compared with other sectors. One of the main reasons of the mistrust shown is the low level of quality of customer service provided an area that generates high costs for the operators themselves, due to the high number of people employed at call centers in order to handle the volume of calls received. To face these challenges, operators launched self-care applications in order to provide customers with a tool that would allow them to autonomously manage the services they have subscribed. In this paper, we present an architecture that provides customized information to customers a solution that is separate from mobile operating systems and communication technologies.
\end{abstract}

Keywords-adaptive interfaces, big data analytics, data mining, user profiling.

\section{Introduction}

Popularity that mobile devices, such as smartphones, enjoy in our society has led to the creation of new types of services available anywhere and anytime [1]. To get an idea of how important these devices have become, we may highlight the huge number of 2.4 billion smartphone users existing worldwide in 2017 [2] an increase of approximately five hundred million users compared to 2015 [3]. This is largely due to the variety of smartphones available on the market [4], and to the multiple services offered by content providers [5], [6]. However, despite the diversity of services that operators offer to their customers, their satisfaction with the telecommunications sector is often below average [7], and a notable number of complaints is reported each year by telecommunication sector regulators [8]. Clients refer to customer service as one of their most significant sources of dissatisfaction [9]. Customer service, is also one of the most significant cost items for operators themselves, due to the number of personnel needed to cope with the large volumes of calls received.

In order to boost customer satisfaction, and also in order to lower the cost associated therewith, telecom operators have introduced self-care applications [10], [11]. In general, self-care applications allow customers to use operators' digital channels to obtain information about their subscribed services, as well as to check account balance, the amount of mobile data used or, promotions offered by operators, to name just a few. As a result, access to customer service becomes more convenient for customers themselves, and operators may take advantage of a new channel to promote their services or products. This means that the potential for tightening ties between operators and customers increases [10].

However, despite all apparent advantages, mobile self-care applications suffer from certain issues that prevent their successful and large-scale use by consumers.

Unoptimized user experience. Many self-care applications made available by service providers suffer from inconsistencies in their navigation flow. For example, some users of Vodafone's self-care application MyVodafone [12] complain about numerous crashes that occur when the app is opening, and report inconsistencies encountered when taking advantage of existing features (e.g. problems with saving pdf invoices, no balance or amount of mobile data used shown). Surveys of self-care applications performed in other countries may be mentioned here as well, with the study described in [13], being one of the examples, where positive and negative aspects of Indian operators' self-care applications are pointed out. The features that affect user experience of these applications include lack of online customer support chats, failure to display information related to recent uploads, continued redirection to external web pages, or inability to add new numbers to the account.

Presentation of poorly customized content. This factor is intrinsically linked to low user experience levels. However, this problem is more far-reaching, since an appealing and well-constructed GUI does necessarily have to present content that is of interest to the user. According to some publications, self-care applications may actually help their users navigate through FAQ lists or surveys, but they provide access to limited, poorly personalized and artificial information [14]. In order to show that they really care about their customers, operators should provide customer support 
chat tools within their self-care applications, as this is one of the communications channels preferred by users [15]. Other issues may be highlighted here as well, such as those related to the presentation of a few or no offers tailored to the user's profile, presentation of inappropriate valueadded services, and scarce information about the services that have been subscribed to [13], [16].

Slow content delivery. Systems used to host all data and traffic are quite complex and often incompatible with today's needs and standards related to access to information. As the co-founder and vice president of Matrixx Software, a provider of digital services for the telecommunications industry, stated in [17], the problem with self-care systems, particularly in the case of large operators, is that they are moving away from real-time charging and billing systems. Thus, a great orchestration of efforts is required to update and retrieve data from, for example, 10 separate systems operating in the background. Thus, it is understandable why many users of self-care applications complain about data loading speeds experienced when using the online solutions [13], [18].

Misuse of customer data. Operators are able to access huge amounts of information about their subscribes. However, much of this information is misused, as it is relied upon to create differentiated services, including the provision of user-oriented products or services within the selfcare applications themselves [19], [20]. In addition, customers' demographic and behavioral data may help create more personalized support mechanisms which, in turn, may increase the degree of satisfaction with operators' customer support services.

To address the issues related to self-care applications that have been mentioned above, the Adaptive Self-care project proposes to research and develop an innovative mobile self-care ecosystem enabling to provide information that is adapted to each customer's profile, via adaptive self-care interfaces. In addition, the ecosystem incorporates a data analysis and management tool to be used by telecom operators, allowing their managers to advertise services and promotional campaigns based on the customer's profile. Individual and anonymous profiles of each customer will be generated by robust big data analytics engines.

In addition to presenting the innovative mobile self-care ecosystem referred to above, this paper also describes the related work related to mobile operators' self-care applications (Section 2), the research methodology used to better define system requirements that need to be complied with (Section 3), and the resulting system architecture (Section 4). The paper is concluded with a discussion about the results obtained and with presentation of potential future work (Section 5).

\section{Related Work}

With the Adaptive Self-care solution presented in this paper, we aim to offer telecommunications operators, as well as their respective customers, an integrated, cloud-based solution that complies with the latest security certifications and standards, and contains mechanisms for big data analytics, machine learning, deep learning, AI, as well as data monetization and governance. The said solution aims to provide operators (via a web application) and customers (via a mobile app) with a number of advantages, such as cost reduction for operators and increased efficiency and customer satisfaction levels for users. However, it needs to be borne in mind that similar mobile self-care solutions, as well as data analysis and management tools are already available on the market. Therefore, this chapter provides a summary of some of those solutions.

The solution offered by Vodafone [12], one of the largest telecommunications operators in Portugal, consists of a web tool and a mobile application adaptable to different screen resolutions of mobile devices, such as smartphones and tablets. It is available for Android and iOS, uses GPS, GSM/4G connectivity and Wi-Fi to enable customers to manage their accounts and services. The app is available in three languages and allows customers to control data consumption, calls and SMS, fill up their accounts and make payment, view invoices, access technical support, receive promotional offers, manage loyalty programs and services, control data and roaming settings and discover all available service. However, personalized content is provided on a very limited scale only.

MyBusiness [21], [22] is an app intended for business customers of Orange, enabling them to manage their companies' telecommunications accounts and data consumption. The app offers the following features: management of all Internet connections, landlines, mobile accounts and data consumption - from one place at any time, management of the company's overall balance, management of account refills and payments, as well as management of multiple accounts and services assigned to specific employees/devices. Despite the features offered, this mobile app is only available for business users and lacks content personalization.

Mitt Telenor [23], Norwegian version of the My Telenor application developed by Telenor Norge, is a mobile customer service application designed by Telenor. The app allows to perform the following activities: buying additional data volumes, managing payments (including the ability to postpone them), managing service subscriptions, locating the nearest Telenor store, making pay-as-you-go payment via Vipps, and accessing customer support services, including SIM card ordering and management, as well as accessing PIN and PUK codes. However, consumers are not provided with access to targeted campaigns advertising products of their interest, which indicates that consumers information is not used to full extent.

The solution adopted by Portuguese telecommunications operator NOS [24], [25] allows customers to manage television, Internet, mobile phone and landline services, namely: balance and data consumption, tariff changes, shipments, access to invoice details from the past six months, access to PIN and PUK codes, as well as access to specific applications of each service. However, nothing is said about 
personalization of content that is provided to customers, e.g. about providing access to targeted marketing campaigns. In addition to mobile self-care applications for telecom customers, we have also identified data management tools and big data analytics platforms available on the market for telecom operators.

Nokia Telecom Analytics [26] is a Nokia solution for big data analytics targeted for telecom operators. It incorporates cognitive data analysis (Nokia's cognitive analytics) methods based on cloud machine deep-learning algorithms that enable real-time monitoring of the data infrastructure, network and connected devices for process optimization and automation, as well as anomaly prevention, and allows to provide optimized customer care services. Despite providing a quality monitoring service, this platform does not make use of consumer data to improve the level of marketing services offered by telecom operators.

Mahindra Comviva's mBAS [27] is a Mahindra Comvivaso solution, widely used on the Indian market. It enables real-time data monetization and offers a distinctive feature that allows to pay for the services provided by the operator or by third parties using the customer's current balance the solution is known as direct carrier billing and enables operators to increase revenue by collaborating with thirdparty digital content providers, such as YouTube and Spotify. It also allows the configuration of generic services and products through a unified platform. No additional information is available on how it uses data provided by consumers to create better offers (services, products, tariffs, etc.).

Calero [28] is an integrated communications lifecycle management (CLM) platform covering landline and mobile lines, as well as mobile data transmission (TDM, MPLS, VoIP and wireless). It aims to provide the operator with a business intelligence solution relying on data analysis to optimize profitability of their business. It automates the process of issuing paper or electronic invoices, facilitates payment processing, including charge allocation, allows to approve payments, traces usage-based internal recoveries, offers detailed multi-currency settlements, audit functions, dispute resolution features (detection of billing errors, downtime, potentially fraudulent activity, misuse/abuse), and provides customer support features, controls the distribution and sharing of services, software licenses and digital assets.

Comarch Telecommunications CEM is Comarch's solution [29] for managing and analyzing the quality of services provided by telecom operators, with a particular focus on increasing customer satisfaction. One of the key requirements for optimizing the experience and the resulting customer satisfaction level of telecom operators is uninterrupted availability of services. Therefore, this solution incorporates an automated fault management tool. This network failure management tool has been adopted by such operators as Vodafone, T-Mobile Poland, Telefonica O2 Germany and MTS Russia, and may be expanded using topological correlation and machine learning techniques to ensure more efficient management of and to prevent net- work failure alarms. The CEM solution integrates the fault management tool with OSS/BSS big data analytics capabilities to provide the operator with real-time monitoring not only of network performance but also of the customers' perception of the network, allowing the operator to take preventive measures in order to guarantee the provision of services and to prevent network failures.

Development of services that are more adequate to consumer preferences in the telecom area is also a research topic for the scientific community. For example, in [30] a study is presented of a new customer segmentation mechanism for the mobile telecommunication sector, based on service usage behaviors. This work relies on data mining technologies deployed for customer clustering and segmentation based on their detail call records and data consumption behaviors. The obtained results are a source of new knowledge about the impact that consumer behavior exerts on their loyalty and satisfaction levels. Other works, such as [31] and [32], also discuss the implementation of advanced techniques for customer segmentation in order to better understand their needs. Nonetheless, neither the scientific community nor the commercial telecom sector have identified a solution that would be capable of both satisfying consumers and telecom operators, since there no standard technique to acquire data concerning consumers in order to optimize the services provided to them has been defined, and no tool or framework capable of monitoring all aspects related to consumers and the network exists as well, which would also allow all services provided by telecom operators to be optimized. Therefore, an intelligent big data management and analytics tool intended for operators, once developed, may prove to be a highly innovative solution for the telecom sector.

\section{Research Methodology}

For the purpose of this project we are using the action-based research methodology that is iterative and user-centered. A session in which customers of telecom operators participated as the target audience for mobile applications that integrate the adaptive self-care ecosystem has been hold. The session took place in three stages: presentation, synergistic debate among the participants and completion of a questionnaire required to conduct a statistical survey concerned with functional requirements and eventual specificities of the mobile application that need to be taken into consideration in the Adaptive Self-care ecosystem. The presentation aimed to introduce the concept of self-care relied upon in the telecommunication operators' systems, which is the main focus of the project. It also served as a point of departure for a synergistic debate, facilitating the alignment of customer interests with the general objectives of the project. Finally, completion of the questionnaire allowed to document, in more detail, the aspects discussed, in order to obtain statistic results allowing to more precisely define the app's priority features. 
Given the statistical results and suggestions obtained via the survey, it was concluded that the session with the participation of customers of telecom operators was beneficial for the project. In addition to proving the clients' demand for such an innovation, it was also possible to set the functional priorities of the application that will contribute, from the end users' point of view, to a higher level of usefulness, flexibility and easier adoption of the system. The analysis of requirements took into account all functionalities proposed in the course of the session, including those considered appropriate and feasible, and enabled to properly adjust those that have already been foreseen in the basic assumptions, to make sure that they comply with, the legal requirements and fit in the context of the project. The goal was to include those features that boost the overall potential of the original project, and not just those that were suggested.

After a comparative analysis of the functionalities offered by commercially available solutions has been performed, and after the study with the individual participants was conducted, it was possible to identify some of the desired functionalities for the Adaptive Self-care solution. This means that both functional and non-functional requirements of the platform could be defined.

\subsection{Functional Requirements}

Functional requirements include those that describe the behavior of the application, i.e. functions or services that the application must be capable of performing. The list below presents the functional requirements applicable to mobile applications intended for customers of telecom operators:

- the application should allow the client to analyze and manage data via lists and graphs, with the filtering option available,

- the application must offer the ability to connect to the Internet via $\mathrm{Wi}-\mathrm{Fi}$ or mobile data,

- the application should be able to collect geographic coordinates using the smartphone's GPS, in order to present location-based content,

- the user must have the ability to add multiple devices to the same account,

- the user must be able to add several accounts to his/her profile (e.g.: 1st party household with Internet, TV, landline, and cell phones of his/her own and their dependents; dependents in a 3rd party household),

- the user should be able to register in the system and edit his/her profile later,

- the application must provide notifications of events/promotions/payment deadlines,

- the user must be able to manage privacy policies,
- the user should be able to locate stores of the operator and of the business partners, using the built-in map,

- the application should provide access to customer service through a chatbot,

- the application must allow to search the operator's products/services,

- the application should allow to subscribe to new services and to purchase products/add-ons.

\subsection{Non-Functional Requirements}

Non-functional requirements are those that relate to application's performance in terms of usability, reliability, security and accuracy of the services offered. The nonfunctional requirements of the Adaptive Self-care mobile application include the following:

- the application interface should be simple and objective,

- the application interface should be flexible, in order to adapt to different resolutions and screen sizes of devices using both Android and iOS operating systems,

- user data should be stored remotely (cloud) and anonymized in order to ensure the privacy of users and of their dependents and, with their consent, the usage of that data by the operator's big data analytics engines which, by its turn, should be capable of enhancing personalized services and promotions,

- the application should be able to submit request responses within 10 seconds. Otherwise, a timeout message should be displayed,

- the database in which non-anonymized user data will be stored should be password protected to prevent unauthorized access,

- Android and iOS applications should be compatible with AI, machine learning, deep learning and big data analytics algorithms operating in the cloud environment,

- compliance with data security and privacy standards applicable to telecommunication services must be ensured.

\section{Results}

Having analyzed different self-care solutions, we propose a cloud infrastructure that satisfies the needs and fills the gaps detected. The central block of the entire system is based on cloud infrastructure. All processing, security and information storage operations are performed here, and the connection between the different applications and external 
systems is ensured. The central block consists of different web services that support mobile applications and backoffice platforms. The cloud infrastructure is also used for creating notifications, collecting and aggregating statistics and routines for consumer profiling, as well as for performing operations that validate business flows. More specifically, the cloud infrastructure comprises the following elements:

- NGINX load balancer [33] which receives and manages all requests, efficiently balancing load and evenly distributing requests across available servers,

- Tomcat server [50] which acts as an application container and allows the implementation and deployment of applications developed using the Java technology,

- MySQL [35], as a database that contains all information stored in a structured way,

- Redis [36], as a non-relational database and data cache,

- Cron jobs which allows tasks to be automatically and periodically scheduled at a pre-set date and time,

- logging system which allows the logging system to be integrated easily, quickly and neatly,

- RESTful web services [37] using the JWT standard for tokenization [38],

- Swagger, as an API development support framework.

The cloud infrastructure of the Adaptive Self-care ecosystem needs to support different types of services, such as storage, business flow processing, business intelligence mechanisms, and smart communication (notification) methodologies. The cloud infrastructure allows the aforementioned services to be integrated, aggregated, customized and delivered to end users based on a distributed and on-demand strategy [39].

\subsection{Architecture}

The cloud infrastructure needs to respond to a number of challenges in order to successfully support the proposed features. Thus, a scalable system needs to be implemented due to the large amount of information that needs to be stored and processed. The system also has to provide forms of communication for the mobile and web applications, including security mechanisms, operator service information processing, consumer profiling algorithm processing, and automated information synchronization tasks.

In order to create an ecosystem that comprises all required components, it has been based on a service-oriented architecture (SOA) model [40]. The SOA architecture model allows to integrate a variety of services that may be exposed or not, depending on the consumer's needs. It also allows to share the services and enables them to communicate with each other. This model also offers flexible integration and cloud web services reusability through a service-based modular architecture, encapsulating several applications and data sources in the form of a black box [41], [42]. The services are exposed based on the RESTful web services [37] standard, i.e. one of the most widely used specifications. REST (Representation State Transfer) is a simpler form of communication than traditional SOAP [43], since the information sent is less detailed and overly complex communications are avoided.

Information is sent in the JSON format [44] that requires less data processing and enables easier interconnection between the services. It is a format-independent technology, since each platform already has its own data interpretation mechanisms. The services are exposed externally through application program interfaces (APIs). APIs are interfaces that enable users to consume cloud services. Development performed outside the APIs is considered part of the software itself, i.e. customer-exposed software [45]. Thus, the project architecture is based on the Software as a Service (SaaS) solution [46]. In SaaS, software is distributed as a service and is implemented natively on one or more web servers. The main goal is to deliver application services to the users as per their requirements [47]. An Internet connection is required to use the services exposed by the software. From the architectural point of view, one may say that SaaS guarantees the following advantages:

- scalable infrastructure - cloud resources may be expanded quickly and cost-effectively, without disrupting the services,

- availability - in accordance with information specified under the previous item, the services are available and accessible anywhere and anytime, thus contributing to greater mobility,

- time and cost savings - it is possible to make the services available to new customers using the same infrastructure, with only a few initial configurations. Such an approach shortens the lead time required to launch the services to new customers and reduces technical costs,

- new features - it is possible to release new features and improvements according to the platform's needs.

\subsection{Information Processing}

In this solution, information is processed in the cloud. Thus, applications (mobile and backoffice) avoid processing huge quantities of data locally. Such an approach is consistent with the line of thought relied upon at all stages of the project development process and allows to avoid various scenarios:

- Slow data processing on the application side. Avoiding complex operations on the users' devices allows to eliminate unexpected errors and ensures smooth operation of the application. If information is processed slowly in the cloud, it is possible to analyze the problem and avoid changing applications. If 
it is a hardware resource issue, cloud-side resource scalability is quite easy to perform due to the nature of the architecture implemented;

- Extreme battery drain. Mobile device battery usage must be taken into account while performing complex processes;

- Security flaws. Communication between applications and the cloud is secure due to the implementation of HTTPS certificates. Thus, sensitive data are only available in the cloud and are not passed outside;

- Bad project development practices. To avoid this issue, mobile/web application developers and backend programmers must observe good project development practices.

\subsection{Data Mining}

The platform uses data mining mechanisms to find useful usage patterns when analyzing data collected by the system. These patterns are relied upon to create user profiles and to automatically adapt their interfaces. Data mining algorithms were applied to acquire knowledge that is based on the data collected, to filter relevant information on the quality of offers and/or discounts, as well as to perform automatic or semi-automatic cataloging and targeting of offers and/or discounts according based on client segmentation. The process of tailoring interfaces to customers must primarily focus on service customization aspects and on quick access to the solution's functionalities. Data mining mechanisms focus on multiple types of customers' behavioral and/or personal data, and they aim to identify information that is most relevant to building consumer profiles and to tailoring the content accordingly.

To achieve the intended objective, the alternating least square (ALS) algorithm was chosen. It is, one of the algorithms that are most frequently used in recommendation systems, and is better known as collaborative filtering (CF). To run the selected algorithm, we used the Spark framework. The intended purpose is to associate the user profile with segments pre-created on the platform (segments may refer, for instance, to product types or tariff types). Information concerning product visualizations and purchases was used. The algorithm relying on this type of data defines product preferences and product types for each consumer and runs through weekly routines to update the results. When the routine commences, the algorithm uses a percentage of the data to create a training file and the remaining percentage to obtain the final output. Once the algorithm is finished, the information is stored in the Redis cache, allowing it to be updated by each user.

When the user checks the suggested products in the mobile application (self-care mobile app), the information is ready to be consumed through the REST web service. To perform the mining task, information about the consumers, i.e. about their behaviors (products that they searched for) and about their purchases is used. A mono-database mining strategy (mining is performed on data sources to identify global interest patterns) was used, as indicated in [48], since the number of data sources needed for the mining task was not high. The main goal of this strategy was to build a recommendation system for clients that would use the self-care mobile app developed under the project. To achieve that purpose, the first step was to group all consumers with similar preferences by using the k-means algorithm $(\mathrm{k}=3)$ and Weka software. Figure 1 presents an example of the data used in the study, containing information about viewed and purchased products. The following fields were taken into consideration: consumer ID, date, time, product ID and product type.

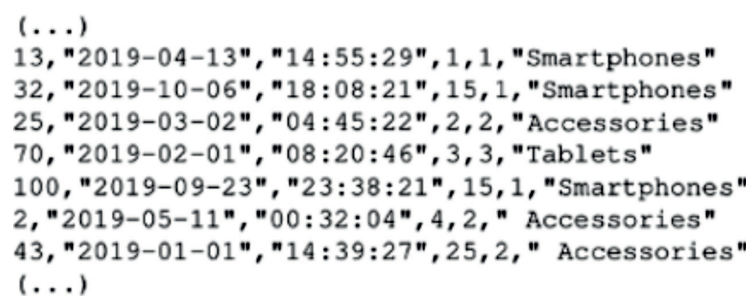

Fig. 1. Example of data used to group all consumers with similar preferences.

Then, clusters were created by using the k-means algorithm. Cluster 0, with $31 \%$ of total data (91 instances), is related to customers who viewed/purchased products within the tablet category. Cluster 1 (47\% of total data - 136 instances) is related to consumers who prefer smartphones. Cluster 2 (22\% of total data - 62 instances) is related to customers whose preferences focus on accessories. Figure 2 depicts the result instances for every cluster. These clusters grouped all consumers based on their purchase preferences. Thus, clients of each segment (cluster) would receive information about campaigns promoting tablets, smartphones or accessories, respectively.

\begin{tabular}{|c|c|c|c|c|}
\hline \multirow{2}{*}{ Atribute } & \multicolumn{5}{|c|}{ Clusters } \\
\cline { 2 - 5 } & 286 & 91 & 136 & 62 \\
\hline Date & $2019-02-08$ & $2019-02-08$ & $2019-03-26$ & $2019-03-26$ \\
\hline Time & $10: 46: 17$ & $10: 46: 17$ & $16: 45: 07$ & $15: 30: 26$ \\
\hline Product & 1 & 6 & 1 & 4 \\
\hline Product type & 1 & 3 & 1 & 2 \\
\hline Class & Smartphones & Tables & Smartphones & Accessories \\
\hline \multicolumn{5}{|c}{} \\
\hline
\end{tabular}

Fig. 2. Clusters created after applying the k-means algorithm.

\subsection{Big Data Analytics}

The prototype aimed to use all information originating from users in order to create a differentiated platform. The main 
concern related to the data structure itself and to the technologies used. In practice, a machine learning tool Apache Spark [42] was used, which basically processes information stored in databases, generates training files (csv), and then processes the saved data output. The framework handles and builds files from resilient distributed datasets (RDD's).

These RDDs may contain any object which operates as a capsule around a small or large dataset, allowing the creation, transformation and invocation of different types of operations within them. The Apache Spark Mlib library allows the usage of operations and algorithms offering fast and high-quality performance parameters in the Java technology, therefore guaranteeing results that are better than those achieved by other solutions available on the market. During this project, different machine learning algorithms were studied, such as: tree methods, logistic regression, linear regression, recommender systems, to name a few, with the ALS recommender system algorithm proving to be the best choice for implementing the project's artificial intelligence solution.

ALS is basically a matrix factorization approach that implements a recommendation algorithm which may be used on e-commerce platforms to recommend products to customers. Unlike some client-based or product-based recommendation systems that calculate similarity, the ALS algorithm uses weight factors, returning a final and justifiable rating from the performed study. In our case, together with the machine learning algorithm, two files were created. The training file will be used, as the name implies, to train the algorithm to obtain more accurate results. The test file, in turn, will return the desired outputs to their APIs. Both files work as a three-phase feedback system. During the first phase, the desired information is collected from the dataset from the training file. In the second phase, where the algorithm uses both files. Relying on the training file, it analyzes customer preferences, based on such attributes as customer ID, the product ID, number of views and purchases made in connection with a given product. Based on that data, the algorithm calculates a percentage ratio and moves on to the next phase. In the third phase, the information is sent to the test file, where it fetches the desired output, either for a particular product or a product type. These files are created in a separate project, where in turn they are stored in a dev environment and updated weekly with customer's actions. For this there are two jobs, allocated in cron job MlClientSegmentProduct, which binds clients to segments and MlRecommendedProduct, which creates the customer recommended product files. In short, the entire process allows customers to be associated with different types of segments, according to their preferences, and to consequently receive product recommendations that are potentially matched to their preferences. A caching system, Redis, has also been implemented to make sure that, in situations where the customer wishes to consult the recommended products, it is not necessary to run the algorithm anew, also making this process faster. A big data analytics mechanism based on a local pattern analytics strategy, such as the one demonstrated in [49], may be used for developing system prototypes, as it may decrease the complexity of the algorithms used to find relevant patterns and may avoid potential data ingestion-related issues.

\subsection{Push Notifications}

As the popularity of smartphones increases, push notifications have become an easy methods used to immediately send information directly to the user's device. Not only do they ensure alert visibility, but they are an important method for maintaining and strengthening customer leads, making them an attractive feature for deploying specific marketing strategies [50], [51]. In Adaptive Self-care, notifications are sent using the Firebase Cloud Messaging (FCM) platform [52]. FCM is one of the components of Google's mobile development platform and supports iOS, Android and web clients. In addition to push notifications, it also sends data messages to be processed by the application. Messages may be intended for three types of targets: a single user, groups of users, or users who have subscribed to specific topics using a given service. As part of the project, several types of push notifications were implemented to provide users with relevant information only. The following are the scenarios adopted for each notification type:

- Limits concerning data, SMS and/or voice usage. The tariffs made available by the operator allow users to enjoy data, voice and SMS. As a rule, each component described above has a predefined limit. Thus, whenever the user exceeds $80 \%$ or $100 \%$ of their limit value, he/she receives a notification. The first notification helps the user get an idea of how much they have spent in the current month. Once the user reaches $100 \%$, they are notified again, and may their access to a given activity/component (data, voice, SMS) may be prevented;

- Generation of a new payment. Users who are postpaid subscribers receive, when an invoice is generated, receive a notification to let them know they have a payment to make. Pre-paid users receive a notification informing them that a given amount has been deducted from their balance or that they have to refill their account to continue enjoying the service;

- New campaign/product. The operator's employees who upload campaigns may trigger a new notification for the system. Using the "create new campaign" form, backoffice users have the option to select segments to which the notification will be sent. Segments are created automatically by the AI module based on the machine learning algorithms that associate users with those segments. This mechanism enables the platform to send targeted notifications to users based on customer profiling;

- Notification on the status of an open report. Using the app, customers may report a problem with their 
service or a bug found in the application. When the operator's employee processes such a report, they can send a notification of the current status of the issue.

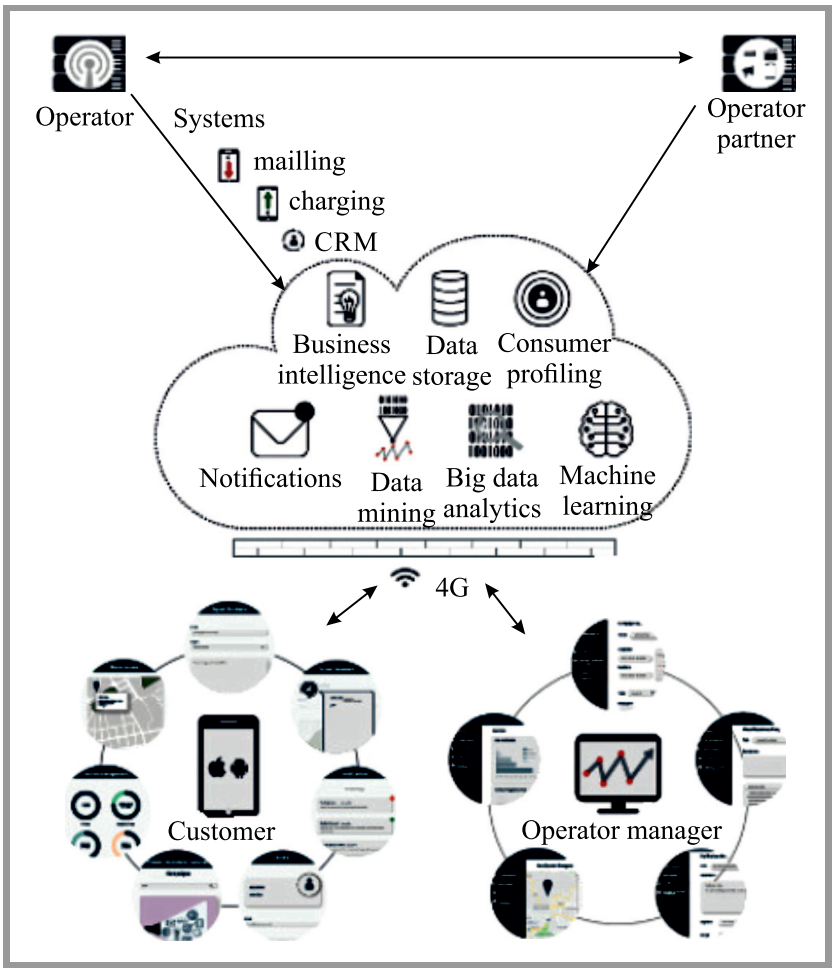

Fig. 3. High-level system architecture.

The notifications that have been sent are stored in the database and their status lets the users know if they have actually been sent or not. When the connection is not available or a service-related problem occurs while sending the notification, a procedure is applied automatically that resends the notification. All data, such as the message body, the content to which it refers (if it is related to campaign, product or payment), the creation date, the actual sending date and the indication whether the user has read it, is stored. Having all notification-related information stored allows the operator to understand whether the notification module is working properly. An API is also available via the web service, allowing users to get all notifications they have received. Such a solution provides the history of notifications via the mobile application. Notifications that have been read are distinguished from ones that remain unread, and their status is marked in the app itself (notification badge).

\section{Conclusion and Future Work}

Our study found gaps in the mobile self-care solutions actually offered by telecom operators. The business management and analytical platforms used by telecom operators also fail to make the best use of consumer data available to them, wasting the opportunity to deliver optimized services and products to consumers. Based on this study, the requirements for the Adaptive Self-care solution were defined, enabling the construction of a cloud architecture that meets those requirements pertaining to storage, processing, scalability and functionalities. For future work, we will do three iterations of the action research methodology, comprising the phases of research, development of the prototype and evaluation (which serves as a point of departure for the next iteration). During the last iteration, the final prototype will be deployed and tested with a specific group of telecom operator customers, as an operational pilot, for the period of one month.

\section{Acknowledgement}

This work is a part of the Adaptive Self-care project, cofunded under COMPETE/P2020/EU, within the framework of the Portuguese Sistema de Incentivos à IDT Empresarial (project 038259).

\section{References}

[1] D. Lamey, "The evolution of technology: past, present and future", Discover Tech, 2018 [Online]. Available:

http://www.discovertec.com/ blog/the-evolution-of-technology (accessed on 2019-11-02)

[2] D. Murphy, "2.4 bn smartphone users in 2017, says eMarketer", Mobile Marketing Magazine, 2017 [Online]. Available: https://mobilemarketingmagazine.com/24bn-smartphone-users-in2017-says-emarketer (accessed on 2019-01-03)

[3] "Number of smartphone users worldwide from 2014 to 2020 (in millions)", Statista [Online]. Available: https://www.statista.com/ statistics/330695/number-of-smartphoneusers-worldwide/

[4] C. Low, "It's no longer enough for budget phones to just be cheap", CES 2017, 2017 [Online]. Available: https://www.engadget.com/ 2017-01-08-budget-phones-ces-2017.html (accessed on 2019-10-04)

[5] "Condiçoes de oferta e de utilizaçao de serviços de comunicaçoes eletrónicas da MEO” [Online]. Available: https://conteudos.meo.pt/ meo/documentos/condicoes-oferta-servicos/mod-c1001276.pdf (accessed on 2019-10-25) [in Portuguese]

[6] D. Nilsson, "Examples of value added services VAS in telecom 2017”, Appland, 2017 [Online]. Available:

https://blog.applandinc.com/examples-mobile-vas-services-vastelecom-2017 (accessed on 2019-02-14)

[7] "Benchmarks by sector", ACSI [Online]. Available: https://www.theacsi.org/acsi-benchmarks/benchmarks-by-sector (accessed on 2019-10-17)

[8] D. de Notícias, "Queixas nas telecomunicales aumentam para 69 mil", 2017 [Online]. Available: https://www.dn.pt/sociedade/ interior/queixas-na-area-das $\ell$ telecomunicacoes-invertem-tendenciae-aumentam-para-69-milem-2016-7205687.html (accessed on 2019-01-04) [in Portuguese]

[9] K. Clarke, "Why mobile operators must break free from bad customer service", Telecom Tech News, 2017 [Online]. Available: https://www.telecomstechnews.com/news/2017/jul/28/whymobileoperators-must-break-free-bad-customer-service/ (accessed on 2019-01-05)

[10] "Mobile self care apps - customer support benefits for telecoms", 2016 [Online]. Available: https://www.kochartech.com/mobile-selfcare-apps-customer-support-benefits-telecoms/ (accessed on 2019-05-05)

[11] "Why seamless self-care should be a top priority for telcos?", Sterlite Tech, 2017 [Online]. Available: https://www.stl.tech/blog/whyseamless-self-care-should-be-a-top-priority-for-telcos/ (accessed on 2019-05-05) 
[12] "My Vodafone Play store web page", 2018 [Online]. Available: https://play.google.com/store/apps/details?id=com.myvodafoneapp (accessed on 2019-02-05)

[13] "Comparison of self-care apps offered by Airtel, Vodafone, Reliance, Rircel, Tata Docomo and BSNL", Telecomtalk, 2016 [Online]. Available: https://telecomtalk.info/comparison-of-selfcare-apps-offeredby-airtel-vodafone-reliance-aircel-tata-docomoand-bsnl/139985/ (accessed on 2019-08-22)

[14] V. Narang, “Telco Dilemma: Prepaid vs Postpaid”, LinkedIn, 2015 [Online]. Available: https://www.linkedin.com/pulse/telco-dilemmaprepaid-vs-postpaid-vaibhav-narang (accessed on 2019-09-20)

[15] V. B. Lestienne, "Digitization and disruption in customer relations", Dimelo, 2015 [Online]. Available: https://www.dimelo.com/en/ blog/digitization-and-disruption-customer-relations/ (accessed on 2019-11-11)

[16] "Users want operators to be more like Amazon, Netflix", Mobile World Live, 2017 [Online]. Available: https://www.mobileworldlive.com/featured-content/top-three/userswant-operators-to-be-more-like-amazon-netflix (accessed on 2019-09-13)

[17] J. Kyriakakis, "The digital enabler: a charging, self-care \& marketing platform at the core of the mobile business", The Black Swan Telecom J., 2016 [Online]. Available: http://bswan.org/digital_ enabler.asp (accessed on 2019-02-05)

[18] "Slow mobile apps ruin everything", New Relic [Online]. Available: https://newrelic.com/resource/slow-mobile-apps-ruin-everything (accessed on 2019-05-10)

[19] A. A. Saunders, "How big data and analytics can drive profitability for telecom operators", Digital Doughnut, 2017 [Online]. Available: https://www.digitaldoughnut.com/articles/2017/august/howbig-dataand-analytics-can-drive-profitability (accessed on 2019-05-07)

[20] H. Prasadh, "By the numbers: three big data opportunities for telecoms", TeleTech, 2013 [Online]. Available: http://www.teletech.com/resources/articles/numbers-three-bigdataopportunities-telecoms (accessed on 2019-05-05)

[21] "MyBusiness aplicaßes on Google Play" [Online]. Available: https://play.google.com/store/apps/details?id=com.evampsaanga. ojoselfcare (accesssed on 2019-08-05)

[22] "My Orange application" [Online]. Available: https:// www.orange.jo/en/services/pages/my-orangeapplication.aspx?utmsource $=$ orangejoutm-medium $=$ linkutmcampaign=homepageutmterm=homepageutm-content=homepage $($ accessed on 2019-06-09)

[23] "Mitt Telenor Google Play webpage" [Online]. Available: https://play.google.com/store/apps/details?id=no.telenor.faktura

[24] “App e rea de Cliente - NOS" [Online]. Available: http://www.nos.pt/particulares/areadecliente/Paginas/default.aspx (accessed on 2019-06-20)

[25] “App NOS Net - NOS” [Online]. Available: http://www.nos.pt/ particulares/internet/internet-fixa/Paginas/app-nos-net.aspx (accessed on 2019-07-03)

[26] "Nokia, Nokia: telecom analytics" [Online]. Available: https://networks.nokia.com/solutions/analytics (accessed on 2019-07-08)

[27] "Subscription platform for telecom operators - Mahindra Comviva" [Online]. Available: https://www.mahindracomviva.com/products/ businesssolutions/centralized-subscription-manager.htm (accessed on 2019-07-18)

[28] "Call accounting reporting software - Calero" [Online]. Available: https://www.calero.com/clm/call-accounting-and-reporting (accessed on 2019-09-22)

[29] "Telecom service assurance: integrated assurance - intelligent assurance analytics - Comarch" [Online]. Available: https://www.comarch.com/telecommunications/serviceassurance/whatis-comarch-integrated-assurance (accessed on 2019-09-20)

[30] S. Aheleroff, "Customer segmentation for a mobile telecommunications company based on service usage behavior", in Proc. 3rd Int. Conf. on Data Mining and Intell. Inf. Technol. App., 2011, pp. $308-313$
[31] J. Bayer, "Customer segmentation in the telecommunications industry”, J. of Database Market. \& Cust. Strat. Manag., vol. 17, no. 3-4, pp. 247-256, 2010 (DOI: 10.1057/dbm.2010.21).

[32] A. Arora and R. Vohra, "Segmentation of mobile customers for improving profitability using data mining techniques", Int. J. Comput. Sc. and Inf. Technol., vol. 5, no. 4, pp. 5241-5244, 2014 [Online]. Available: http://ijcsit.com/docs/Volume\%205/vol5issue04/ ijcsit2014050492.pdf

[33] NGINX webpage [Online]. Available: https://www.nginx.com/

[34] Apache Tomcat webpage [Online]. Available: http://tomcat.apache.org/

[35] MySQL webpage [Online]. Available: https://www.mysql.com/ (accessed on 2019-09-15)

[36] Redis webpage [Online]. Available: https://redis.io/ (accessed on 2019-08-13)

[37] "RESTful Web services", IBM Developer [Online]. Available: https://developer.ibm.com/articles/ws-restful/

[38] JSON web token [Online]. Available: https://jwt.io/

[39] R. Nagarajan and R. Thirunavukarasu, "A review on intelligent cloud broker for effective service provisioning in cloud" in Proc. of the 2nd Int. Conf. on Intell. Comput. and Control Syst., 2018 (DOI: 10.1109/ICCONS.2018.8662953).

[40] D. K. Barry, "Service oriented architecture (SOA)", Barry Associates [Online]. Available: https://www.servicearchitecture.com/ articles/web-services/service-oriented-architecturesoadefinition.html (accessed on 2019-09-10)

[41] N. Niknejad, W. Ismail, I. Ghani, B. Nazari, M. Bahari, and A. Hussin, "Understanding service-oriented architecture (SOA): a systematic literature review and directions for further investigation”, Inf. Systems, vol. 91, 2020 (DOI: 10.1016/j.is.2020.101491).

[42] R. Nagarajan and R. Thirunavukarasu, "Service-oriented broker for effective provisioning of cloud services - a survey", Int. J. of Comput. and Dig. Syst., vol. 9, no. 5, pp. 863-879, 2020 (DOI: 10.12785/ijcds/090508).

[43] "SOAP version 1.2 part 1: messaging framework (second edition)" [Online]. Available: https://www.w3.org/TR/soap12/ (accessed on 2019-09-14)

[44] "Introducing JSON" [Online]. Available: https://www.json.org (accessed on 2019-04-02)

[45] J. Freeman, "What is an API? Application programming interfaces explained", InfoWorld, 2019 [Online]. Available: https://www.infoworld.com/article/3269878/what-is-an-apiapplication-programming-interfaces-explained.html (accessed on 2019-09-10)

[46] "Service-oriented architecture (SOA)", IBM [Online]. Available: https://www.ibm.com/support/knowledgecenter/en/SSMQ79_9.5.1/ com.ibm.egl.pg.doc/topics/pegl_serv_overview.html (accessed on 2019-09-10)

[47] N. Rajganesh and T. Ramkumar, "A review on broker based cloud service model", J. of Comput. and Inf. Technol., vol. 24, no. 3, pp. 283-292, 2016 (DOI: 10.20532/cit.2016.1002778).

[48] T. Ramkumar, S. Hariharan, and S. Selvamuthukumaran, "A survey on mining multiple data sources", Wiley Interdiscip. Rev. Data Min. and Knowl. Discov., vol. 3, no. 1, pp. 1-11, 2013 (DOI: 10.1002/widm.1077).

[49] T. Venkatesan, K. Saravanan, and T. Ramkumar, "A big data recommendation engine framework based on local pattern analytics strategy for mining multi-sourced big data", J. of Inform. \& Knowl. Manag., vol. 18, no. 1, 2019 (DOI: 10.1142/S0219649219500096).

[50] "Apache Spark webpage" [Online]. Available: https://spark.apache.org (accessed on 2019-04-27)

[51] S. Anquetil, "What are push notifications and why are they important to your app?", Business 2 Community, 2014 [Online]. Available: https://www.business2community.com/mobileapps/push-notifications-important-app-01008413

[52] "Firebase cloud messaging", Firebase Google [Online]. Available: https://www.firebase.google.com/docs/cloud-messaging/ (accessed on 2019-07-15) 


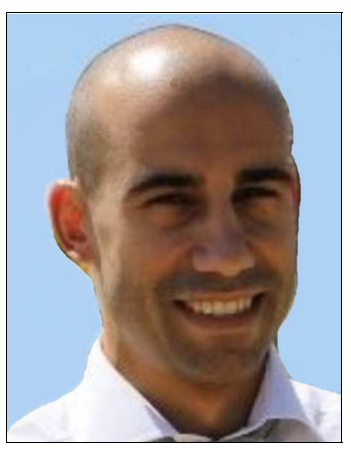

Patrick Santos is working as a backend developer at TIMWE SPIN Lab. He has received his M.Sc. degree in Computer Science from the Technology High School, Castelo Branco, Portugal. He has also contributed with some scientific publications on multiple subjects, such as context-aware systems, intelligent personal assistants, and payment ecosystems. His main research interests include ubiquitous computing and Big Data.

E-mail: patrick.santos@timwetech.com

TIMWE Spin Lab

Parque da Ciencia e da Tecnologia da Universidade do Porto

Porto, Portugal

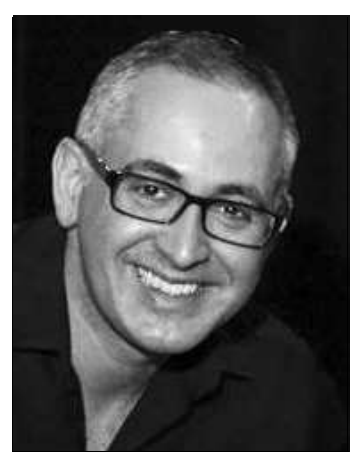

Jorge Ramos is currently working as a Project Manager at TIMWE SPIN Lab, a company in Porto, Portugal. He has a B.Sc. degree in Electrical Engineering and Computer Science from Nova University in Lisbon. Jorge has considerable experience in project management in the telecommunications sector, thanks to more than 15 years spent working with telco operators. His research interests include network virtualization techniques and delay tolerant networks.

E-mail: jorge.ramos@timwetech.com

TIMWE Spin Lab

Parque da Ciencia e da Tecnologia da Universidade do Porto

Porto, Portugal

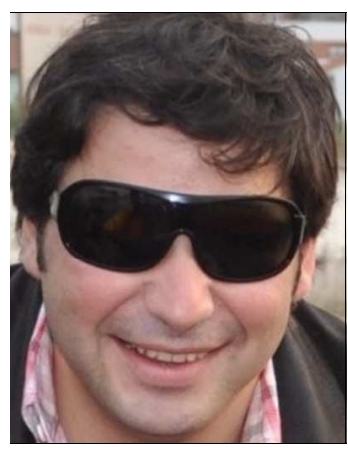

Eduardo Seabra is working at TIMWE SPIN Lab as a backend developer. He received his M.Sc. in Electronic Engineering and Telecommunications from the University of Aveiro, Portugal. He was a researcher at the Instituto de Telecomunicações, with his main research interests focusing on Web 2.0 and IPTV services.

E-mail: eduardo.seabra@timwetech.com

TIMWE Spin Lab

Parque da Ciencia e da Tecnologia da Universidade do Porto

Porto, Portugal

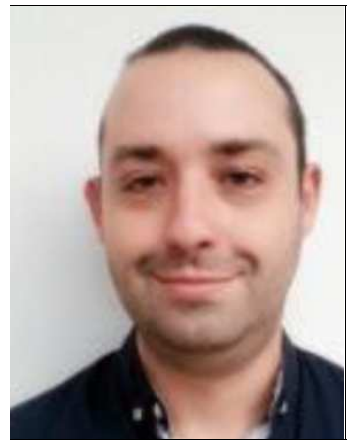

José Castro is currently working at TIMWE SPIN Lab as a frontend developer. He has an M.Sc. in Information Systems Engineering and Management from the University of Minho, Portugal. José boasts considerable experience in developing mobile solutions (Android and iOS) and also in developing web platforms for customers from different sectors. His main research interests include distributed systems and computer security.

E-mail: jose.castro@timwetech.com

TIMWE Spin Lab

Parque da Ciencia e da Tecnologia da Universidade do Porto

Porto, Portugal 